PROCEEDINGS OF THE

AMERICAN MATHEMATICAL SOCIETY

Volume 128, Number 11, Pages 3329-3334

S 0002-9939(00)05763-4

Article electronically published on May 11, 2000

\title{
COMMUTATORS FOR THE MAXIMAL AND SHARP FUNCTIONS
}

\author{
JESÚS BASTERO, MARIO MILMAN, AND FRANCISCO J. RUIZ
}

(Communicated by Christopher D. Sogge)

\begin{abstract}
The class of functions for which the commutator with the HardyLittlewood maximal function or the maximal sharp function are bounded on $L^{q}$ are characterized and proved to be the same.
\end{abstract}

For the Hilbert transform $H$, and other classical singular integral operators, a well known and important result due to Coifman, Rochberg and Weiss (cf. [2]) states that a locally integrable function $b$ in $\mathbb{R}^{n}$ is in $B M O$ if and only if the commutator $[H, b]$, defined by

$$
[H, b] f=H(b f)-b H(f),
$$

is bounded in $L^{q}$, for some (and for all) $q \in(1, \infty)$. The cancellation implied by the commutator operation and the properties of singular integrals are crucial for the validity of the result. Later in 3], using real interpolation techniques, Milman and Schonbeck proved a commutator result that applies to the Hardy-Littlewood maximal operator $M$ as well as the sharp maximal operator. In fact the commutator result is valid for a large class of nonlinear operators which we now describe.

Let us say that $T$ is a positive quasilinear operator if it is defined on a suitable class of locally integrable functions $D(T)$ and satisfies

i) $T f \geq 0$, for $f \in D(T)$,

ii) $T(\alpha f)=|\alpha| T f$, for $\alpha \in \mathbb{R}$ and $f \in D(T)$,

iii) $|T f-T g| \leq T(f-g)$, for $f, g \in D(T)$.

We have (cf. [3])

Proposition 1. Let $b$ be a nonnegative BMO function and suppose that $T$ is a positive quasilinear operator which is bounded on $L^{q}(w)$, for some $1 \leq q<\infty$ and for all $w$ weights belonging to the Muckenhoupt class $\mathcal{A}_{r}$ for some $r \in[1,+\infty)$. Then $[T, b]$ is bounded on $L^{q}$.

In particular the result applies to the maximal operator and the sharp maximal function. Note that since the Hardy-Littlewood maximal operator $M$ is a positive

Received by the editors January 6, 1999 .

2000 Mathematics Subject Classification. Primary 42B25, 46E30.

Key words and phrases. Maximal functions, sharp function, BMO, commutators.

The first author was partially supported by DGES.

The second author's research was partially supported by the "Comisión de Doctorado" from the University of Zaragoza during his visit there.

The third author was partially supported by DGES and UR.

(C)2000 American Mathematical Society 
operator, the positivity condition on $b$ seems crucial to effect the necessary cancellation. In fact, the closed graph theorem implies that if $b$ is a negative locally integrable function, then $[M, b]$ is bounded on $L^{q}$ for some $q \in(1, \infty)$ if and only if $b \in L^{\infty}$, since in this case we have

$$
M(b f)-b M(f) \geq|b| M(f) \geq|b f| .
$$

However, the question of obtaining a complete characterization of commutators with the Hardy-Littlewood maximal operator in the spirit of the Coifman-RochbergWeiss theorem has apparently remained open. In this note we show that a slightly extended form of positivity is a necessary and sufficient condition to characterize the boundedness of $[M, b]$. To see what this condition should be we observe that if $M$ were a linear operator, then, given that everything we do is modulo bounded operators, the correct requirement would appear to be that $b \in B M O$ with its negative part $b^{-}$bounded. Indeed, the sufficiency of the condition $b \in B M O$ with $b^{-}$bounded formally follows from Proposition 1, the fact that $b \in B M O \Rightarrow|b| \in$ $B M O$ and the estimate

$$
|[M, b] f-[M,|b|] f| \leq 2\left(b^{-} M(f)\right) .
$$

We summarize the previous discussion with the following

Proposition 2. If $b$ is a BMO function such that its negative part $b^{-}$is bounded, then the commutator $[M, b]$ is bounded on $L^{q}$, for all $q \in(1, \infty)$.

The purpose of this note is to prove the converse of Proposition 2 and to show that a similar characterization also holds for the sharp maximal operator.

Let us formally note that the proof of Proposition 1 given in [3] implies the following more general result

Proposition 3. Let $b$ be a function in $B M O$, with $b^{-} \in L^{\infty}$ and let $T$ be a positive quasilinear operator, which is bounded on $L^{q}(w)$, for some $1 \leq q<\infty$ and for all $w$ weights belonging to the Muckenhoupt class $\mathcal{A}_{r}$ for some $r \in[1,+\infty)$. Then $[T, b]$ is bounded on $L^{q}$.

Proof. Note that the following variant of (1) holds for any positive quasilinear operator $T$ :

$$
|[T, b] f-[T,|b|] f| \leq 2\left(b^{-} T(f)+T\left(b^{-} f\right)\right) .
$$

Therefore, since the right hand side is bounded, the result follows immediately from Proposition 1.

Remark. Since it could be useful for other purposes we note that an alternative proof of Proposition 3 can be given by following the proof given in 3 verbatim and observing that, under the assumption that $b^{-}$is bounded, the pair $\left(L^{q}\left(e^{b}\right), L^{q}\left(e^{-b}\right)\right)$ is ordered.

Recall that for a locally integrable function and for $1 \leq p<\infty$, the HardyLittlewood maximal function is defined by

$$
M_{p}(f)(x)=\sup _{x \in Q}\left(\frac{1}{|Q|} \int_{Q}|f|^{p}\right)^{1 / p}
$$

for all $x \in \mathbb{R}^{n}$, where $Q$ denotes a cube with sides parallel to the coordinate axes and $|Q|$ the Lebesgue measure of $Q$. Note that for $p=1, M_{p}=M$ is the classical 
Hardy-Littlewood maximal operator. The sharp function is given by

$$
f^{\sharp}(x)=M^{\sharp} f(x)=\sup _{x \in Q} \frac{1}{|Q|} \int_{Q}\left|f-f_{Q}\right|, \quad x \in \mathbb{R}^{n},
$$

where as usual we let $f_{Q}=\frac{1}{Q} \int_{Q} f$.

If $Q_{0}$ is a fixed cube, then the Hardy-Littlewood maximal function relative to $Q_{0}$ is given by

$$
M_{p, Q_{0}}(f)(x)=\sup _{x \in Q \subseteq Q_{0}}\left(\frac{1}{|Q|} \int_{Q}|f|^{p}\right)^{1 / p}
$$

defined for all $x \in Q_{0}$.

Our main result for $M_{p}$ can be now stated as follows

Proposition 4. Let $b$ be a real valued, locally integrable function in $\mathbb{R}^{n}$. The following assertions are equivalent:

(4.1) The commutator $\left[M_{p}, b\right]$ is bounded in $L^{q}$, for all $q, p<q<\infty$.

(4.2) The commutator $\left[M_{p}, b\right]$ is bounded in $L^{q}$, for some $q, p<q<\infty$.

(4.3) $b$ is in $B M O$ and $b^{-}$is in $L^{\infty}$.

(4.4) There exists $q \in[1, \infty)$ such that

$$
\sup _{Q} \frac{1}{|Q|} \int_{Q}\left|b-M_{p, Q} b\right|^{q}<\infty .
$$

(4.5) For all $q \in[1, \infty)$ we have

$$
\sup _{Q} \frac{1}{|Q|} \int_{Q}\left|b-M_{p, Q} b\right|^{q}<\infty .
$$

Proof. Since the implications (4.1) $\Rightarrow(4.2)$ and (4.5) $\Rightarrow$ (4.4) follow readily, we only have to prove (4.3) $\Rightarrow(4.1),(4.2) \Rightarrow(4.4)$ and $(4.4) \Rightarrow(4.3)$ (the implication $(4.1) \Rightarrow(4.5)$ is similar to $(4.2) \Rightarrow(4.4))$.

$(4.3) \Rightarrow(4.1)$. The conclusion follows from Proposition 3 and the fact that $M_{p}$ is bounded in $L^{q}(w)$ for all $w$ in the class $\mathcal{A}_{q / p}$.

$(4.2) \Rightarrow(4.4)$. We consider $f=\chi_{Q} \in L^{q}$. Then

$$
\left(\int_{Q}\left|M_{p}(b f)-b M_{p}(f)\right|^{q}\right)^{1 / q} \leq\left\|\left[M_{p}, b\right] f\right\|_{q} \leq C\|f\|_{q}=C|Q|^{1 / q}
$$

implies the result, since $M_{p, Q}\left(\chi_{Q}\right)=\chi_{Q}$ and $M_{p}\left(b \chi_{Q}\right)(x)=M_{p, Q}(b)(x)$ for all $x \in Q$.

$(4.4) \Rightarrow(4.3)$. Let $Q$ be a fixed cube. By hypothesis and Hölder's inequality we have

$$
\frac{1}{|Q|} \int_{Q}\left|b-M_{p, Q} b\right| \leq\left(\frac{1}{|Q|} \int_{Q}\left|b-M_{p, Q} b\right|^{q}\right)^{1 / q} \leq C .
$$

Let $E=\left\{x \in Q ; b(x) \leq b_{Q}\right\}$ and $F=\left\{x \in Q ; b(x)>b_{Q}\right\}$. The following equality is trivially true:

$$
\int_{E}\left|b-b_{Q}\right|=\int_{F}\left|b-b_{Q}\right|
$$


Then

$$
\begin{aligned}
\frac{1}{|Q|} \int_{Q}\left|b-b_{Q}\right| & =\frac{2}{|Q|} \int_{E}\left|b-b_{Q}\right| \leq \frac{2}{|Q|} \int_{E}\left|b-M_{p, Q}(b)\right| \\
& \leq \frac{2}{|Q|} \int_{Q}\left|b-M_{p, Q}(b)\right| \leq C
\end{aligned}
$$

since for $x \in E$ we have $b(x) \leq b_{Q} \leq M_{p, Q}(b)(x)$ and so we achieve $b \in B M O$.

In order to show show that $b^{-} \in L^{\infty}$, observe that $M_{p, Q}(b) \geq|b|$ in $Q$ and therefore we have that, in $Q$,

$$
0 \leq b^{-} \leq M_{p, Q} b-b^{+}+b^{-}=M_{p, Q}(b)-b .
$$

Combining estimates we see that there exists a constant $C$ such that for any cube $Q$ we have

$$
\left(b^{-}\right)_{Q} \leq C .
$$

Thus, the boundedness of $b^{-}$follows from Lebesgue's differentiation theorem.

It can be readily seen that a local version of our main result also holds. More precisely, if we fix a cube $Q$ and consider the commutator $\left[M_{Q}, b\right]$ the analog of Proposition 4 holds with the obviously implied localized conditions. For example, if $b$ is a $B L O(Q)$ function (cf. [1]), then it automatically satisfies $b^{-} \in L^{\infty}(Q)$. In fact if $b \in B L O(Q)$, then for a suitable constant $c$, we have

$$
b_{Q} \leq \inf _{Q} b+c .
$$

Therefore, we have the following

Corollary 5. Let $b \in B L O(Q)$ and $q \in(1, \infty)$. Then the commutator $\left[M_{Q}, b\right]$ is bounded on $L^{q}(Q)$.

Remark. It could be worthwhile to point out the connection between $B L O$ and the class of functions that satisfy the conditions of Proposition 4. In fact it is well known (cf. [1) that an integrable positive function $b \in B L O(Q)$ iff $M_{Q}(b)-b$ is bounded. The reader should compare this with condition (4.5) above. More generally, an integrable function $b$ belongs to $B L O(Q)$ if and only if there exist $g \in B M O(Q)$ and $h \in L^{\infty}(Q)$ such that $b=M_{Q} g+h$ (cf. [1]). Let us give another proof of Corollary 5 based on this representation. Note that since $g \in$ $B M O(Q)$, we have $M_{Q} g \in B L O(Q)$ (cf. [1]) and moreover since $M_{Q} g$ is positive, we have that $\left[M_{Q}, M_{Q} g\right]$ is bounded on $L^{q}$ by Proposition 4 . It is readily seen that $[M, b]-\left[M, M_{Q} g\right]$ is bounded on $L^{q}$ and therefore the boundedness of $[M, b]$ follows. These comments suggest that the class of functions singled out in Proposition 4 could be of interest in other contexts.

Next we consider commutators with the sharp function. The results are similar to those in Proposition 4.

Proposition 6. Let $b$ be a real valued, locally integrable function in $\mathbb{R}^{n}$. The following assertions are equivalent:

(6.1) The commutator $\left[M^{\sharp}, b\right]$ is bounded in $L^{q}$ for all $q, 1<q<\infty$.

(6.2) The commutator $\left[M^{\sharp}, b\right]$ is bounded in $L^{q}$ for some $q, 1<q<\infty$.

(6.3) $b$ is in $B M O$ and $b^{-}$is in $L^{\infty}$. 
(6.4) There exists $q \in[1, \infty)$ such that

$$
\sup _{Q} \frac{1}{|Q|} \int_{Q}\left|b(x)-2\left(b \chi_{Q}\right)^{\sharp}(x)\right|^{q} d x<\infty .
$$

(6.5) For all $q \in[1, \infty)$ we have

$$
\sup _{Q} \frac{1}{|Q|} \int_{Q}\left|b(x)-2\left(b \chi_{Q}\right)^{\sharp}(x)\right|^{q} d x<\infty .
$$

Proof. $(6.3) \Rightarrow(6.1)$. It follows from Proposition 3 , since for all locally integrable functions $f$ we have $M^{\sharp} f \leq 2 M(f)$ and the weighted estimates are guaranteed.

$(6.2) \Rightarrow(6.4)$. Let $Q$ be a fixed cube as before. Let $Q_{1}$ be another cube; it is easy to compute that

$$
\frac{1}{\left|Q_{1}\right|} \int_{Q_{1}}\left|\chi_{Q}-\left(\chi_{Q}\right)_{Q_{1}}\right|=\frac{2\left|Q_{1} \backslash Q\right|\left|Q_{1} \cap Q\right|}{\left|Q_{1}\right|^{2}} \leq \frac{1}{2}
$$

(recall that for nonnegative numbers $4 r s \leq(r+s)^{2}$ ). On the other hand, given $x \in Q$ there always exists a cube $Q_{1}$ containing $Q$ such that $\left|Q_{1}\right|=2|Q|$. This shows that $\left(\chi_{Q}\right)^{\sharp}(x)=1 / 2$, for all $x \in Q$. Hence the conclusion follows readily as in Proposition 4.

$(6.4) \Rightarrow(6.3)$. We proceed as in the corresponding portion of the proof of Proposition 4 , but some extra difficulties appear.

First, our claim is to prove that

$$
\left|b_{Q}\right| \leq 2\left(b \chi_{Q}\right)^{\sharp}(x), x \in Q .
$$

Let $x \in Q$ and pick a cube $Q_{1}$ containing $Q$ and with volume $\left|Q_{1}\right|=2|Q|$. Then

$$
\begin{aligned}
\left(b \chi_{Q}\right)^{\sharp}(x) & \geq \frac{1}{\left|Q_{1}\right|} \int_{Q_{1}}\left|b \chi_{Q}(y)-\left(b \chi_{Q}\right)_{Q_{1}}\right| d y \\
& =\frac{1}{2|Q|}\left(\int_{Q}\left|b(y)-\frac{1}{2} b_{Q}\right| d y+\frac{1}{2}\left|Q_{1} \backslash Q\right|\left|b_{Q}\right|\right) \\
& =\frac{1}{2|Q|} \int_{Q}\left|b(y)-\frac{1}{2} b_{Q}\right| d y+\frac{1}{4}\left|b_{Q}\right| .
\end{aligned}
$$

On the other hand

$$
\begin{aligned}
\left|b_{Q}\right| & \leq \frac{1}{|Q|} \int_{Q}\left|b(y)-\frac{1}{2} b_{Q}\right| d y+\frac{1}{|Q|} \int_{Q}\left|\frac{1}{2} b_{Q}\right| d y \\
& =\frac{1}{|Q|} \int_{Q}\left|b(y)-\frac{1}{2} b_{Q}\right| d y+\frac{1}{2}\left|b_{Q}\right|,
\end{aligned}
$$

and so

$$
\frac{1}{2}\left|b_{Q}\right| \leq \frac{1}{|Q|} \int_{Q}\left|b(y)-\frac{1}{2} b_{Q}\right| d y .
$$

Finally, (3) and (4) lead us to (2). 
We can now achieve that $b \in B M O$. Indeed, let $E=\left\{x \in Q ; b(x) \leq b_{Q}\right\}$. Then

$$
\begin{aligned}
\frac{1}{|Q|} \int_{Q}\left|b(x)-b_{Q}\right| d x & =\frac{2}{|Q|} \int_{E}\left(b_{Q}-b(x)\right) d x \\
& \leq \frac{2}{|Q|} \int_{E}\left(2\left(b \chi_{Q}\right)^{\sharp}(x)-b(x)\right) d x \\
& \leq \frac{2}{|Q|} \int_{E}\left|2\left(b \chi_{Q}\right)^{\sharp}(x)-b(x)\right| d x \\
& \leq \frac{2}{|Q|} \int_{Q}\left|2\left(b \chi_{Q}\right)^{\sharp}(x)-b(x)\right| d x \leq C,
\end{aligned}
$$

where we have applied (2) and the hypothesis.

In order to prove that $b^{-} \in L^{\infty}$ we also use (2). We start from the inequality

$$
2\left(b \chi_{Q}\right)^{\sharp}(x)-b(x) \geq\left|b_{Q}\right|-b^{+}(x)+b^{-}(x), x \in Q .
$$

Averaging on $Q$, we have

$$
\begin{aligned}
C & \geq \frac{1}{|Q|} \int_{Q}\left|2\left(b \chi_{Q}\right)^{\sharp}(x)-b(x)\right| d x \\
& \geq \frac{1}{|Q|} \int_{Q}\left(2\left(b \chi_{Q}\right)^{\sharp}(x)-b(x)\right) d x \\
& \geq \frac{1}{|Q|} \int_{Q}\left(\left|b_{Q}\right|-b^{+}(x)+b^{-}(x)\right) d x \\
& =\left|b_{Q}\right|-\frac{1}{|Q|} \int_{Q} b^{+}(x) d x+\frac{1}{|Q|} \int_{Q} b^{-}(x) d x .
\end{aligned}
$$

Letting $|Q| \rightarrow 0$, with $x \in Q$, Lebesgue differentiation theorem assures that

$$
C \geq|b(x)|-b^{+}(x)+b^{-}(x)=2 b^{-}(x)
$$

and the desired result follows.

The remaining proofs are similar to the ones in Proposition 4 and we leave the details to the interested reader.

\section{REFERENCES}

1. C. Bennett and R. Sharpley, Interpolation of operators Academic Press, 1988. MR 89e:46001

2. R. Coifman, R. Rochberg and G. Weiss, Factorization theorems for Hardy spaces in several variables Ann. Math. 103 (1976), 611-635. MR 54:843

3. M. Milman and T. Schonbek Second order estimates in interpolation theory and applications Proc. Amer. Math. Soc. 110 (4) 1990, 961-969. MR 91k:46088

Department of Mathematics, University of Zaragoza, 50009-Zaragoza, Spain

E-mail address: bastero@posta.unizar.es

Department of Mathematics, Florida Atlantic University, Boca Raton, Florida 33431

E-mail address: interpol@bellsouth.net

Department of Mathematics, University of Zaragoza, 50009-Zaragoza, Spain

E-mail address: fjruiz@posta.unizar.es 